\title{
A RE-EMERGÊNCIA DA COQUELUCHE: DA ROTINA DOS ATENDIMENTOS AO IMPERATIVO DA BIOSSEGURANÇA
}

\author{
Flávio Rocha da Silvale 2, Marli Brito M. de Albuquerque Navarro 3 , Bernardo E. \\ Correa Soares $^{2}$ e Salvatore Giovanni De-Simone 4 es
}

\section{RESUMO}

Dados recentes mostram que, no Brasil, foram notificados, no ano de 2012, 15.428 casos suspeitos de coqueluche, dos quais 4.453 (28,9\%) foram confirmados, o que representa um aumento de $97 \% \mathrm{em}$ relação ao mesmo período de 2011, ano em que foram registrados 2.258 casos da doença. Crianças com menos de 1 ano de idade apresentam taxas de incidência e letalidade mais acentuadas. Este estudo teve como objetivo identificar a percepção dos profissionais de saúde atuantes nas unidades de saúde do Estado do Rio de Janeiro no atendimento a pacientes com suspeita ou confirmação de doenças respiratórias, como coqueluche e tuberculose, a adoção das práticas de biossegurança e a existência de programa de capacitação continuada. Buscamos recolher informações referentes à exposição ao risco de contaminação, observando o aspecto subjetivo da percepção do profissional. Estas informações foram obtidas por meio de questionários aplicados em unidades de atendimento situadas no Estado do Rio de Janeiro. A metodologia aplicada ao estudo privilegiou o aspecto qualitativo por ser mais adequado às análises de percepção, nas quais o aspecto subjetivo adquire relevância. Os resultados demonstraram que o aprimoramento profissional nas unidades de saúde por meio de investimento em programa de capacitação continuada nas temáticas biossegurança e doenças respiratórias, como coqueluche e tuberculose, é fundamental para reverter a situação encontrada nesta pesquisa, bem como o maior investimento em infraestrutura nas unidades de saúde, especialmente nas unidades municipais.

DESCRITORES: Coqueluche; biossegurança; unidade de saúde; tuberculose; Rio de Janeiro.

1 Instituto Oswaldo Cruz, Fundação Oswaldo Cruz, Rio de Janeiro, RJ, Brasil.

2 Instituto de Pesquisa Clínica Evandro Chagas, Fundação Oswaldo Cruz, Rio de Janeiro-RJ, Brasil.

3 Núcleo de Biossegurança-Escola Nacional de Saúde Pública, Fundação Oswaldo Cruz, Rio de Janeiro-RJ, Brasil.

4 Centro de Desenvolvimento Tecnológico em Saúde (CDTS)/ Instituto Nacional de Ciência e Tecnologia de Inovação em Doenças Negligenciadas (INCT-IDN), Fundação Oswaldo Cruz, Rio de Janeiro, RJ, Brasil.

5 Departamento de Biologia Celular e Molecular, Universidade Federal Fluminense, Niterói, Rio de Janeiro, Brasil

Endereço para correspondência: Flávio Rocha da Silva. E-mail: flavio.rocha@ioc.fiocruz.br

Recebido para publicação em: 22/4/2013. Revisto em: 3/2/2014. Aceito em: 10/2/2014. 


\begin{abstract}
The re-emergence of Pertussis: from routine healthcare to the need for biosafety actions

In Brazil in the year 2012, 15,428 suspected pertussis cases were reported, from which 4,453 (28.9\%) were confirmed, representing an increase of $97 \%$ over the same period in 2011, when 2,258 cases of pertussis were confirmed. Children under one year of age belonged to the group with more pronounced rates of incidence and mortality. The study had the objective of establishing the perception of health professionals in health facilities in the state of Rio de Janeiro among patients attending with suspected or confirmed respiratory diseases such as whooping cough and tuberculosis, and the adoption of biosafety practices and continued training programs. The methodology used in the study favored the qualitative aspect, since it has been better suited to analysis of perception where the subjective aspect would be considered relevant. The research results showed that professional improvement in health facilities by investing in ongoing training programs on biosafety issues and respiratory diseases such as whooping cough and tuberculosis, is an essential issue to reverse this situation. Research, and large investments in infrastructure of health units, especially in the municipal hospitals, is most needed.
\end{abstract}

KEY WORDS: Pertussis; biosafety; health unit; tuberculosis; Rio de Janeiro, Brazil.

\title{
INTRODUÇÃO
}

A contaminação por patógenos nos locais de atendimento à saúde, especialmente nos hospitais e naqueles em que são realizadas as emergências, tem se caracterizado como fenômeno recorrente. A complexidade da infecção hospitalar representa, pois, um desafio para as instituições de saúde $(1,2,3,4,6,11)$.

Tal complexidade abrange a saúde dos profissionais, o controle das infecções entre os pacientes e o monitoramento do ambiente como contexto relacionado à implementação de políticas institucionais de biossegurança visando, prioritariamente, o desenvolvimento de uma cultura de prevenção $(1,3,4,6,11)$.

A deficiência de ações preventivas voltadas para monitoramentos eficazes de risco vulnerabiliza, em maior ou menor grau, a totalidade dos profissionais que atuam nos estabelecimentos de saúde e também as pessoas que circulam nestes locais, como pacientes, acompanhantes e visitantes $(1,4,6,11)$.

Segundo Nichiata et al. (2004),

[...] a adesão às ações de biossegurança constitui o maior desafio, desde as primeiras formulações das medidas de proteção, inicialmente referidas como isolamentos. A despeito do desenvolvimento e emprego da tecnologia dos materiais utilizados em serviços de saúde, em especial os descartáveis e das técnicas e procedimentos recomendados na prevenção das infecções e das doenças transmissíveis, não se tem conseguido a redução das ocorrências das infecções e das doenças entre os trabalhadores. 
A contaminação por Bordetella pertussis, por exemplo, pode expandir-se rapidamente e sair das emergências, contaminando os Centros de Terapia Intensiva, as enfermarias e pacientes imunocomprometidos, as maternidades, os berçários e os setores pediátricos, o que caracteriza um quadro de expressivo risco $(7,8,9,10)$. Isso pode ocorrer quando não há a identificação correta da coqueluche, realizada clinicamente e/ou por exame laboratorial, o que servirá de base para as medidas de isolamento com sentido preventivo do processo de contaminação.

Potencialmente perigosa, a coqueluche caracteriza-se como doença infecciosa aguda, altamente contagiosa. Extremamente virulenta, o contágio se realiza pela propagação das gotículas nasofaríngeas quando o doente tosse, espirra e fala. É importante realçar que, até 1940, era a maior causa de mortalidade infantil no mundo. Apesar de ser uma doença imunoprevenível, em 2001, a World Health Organization (WHO) revelava a ocorrência anual, em nível global, de 50 milhões de casos, registrando mais de 300 mil mortes $(1,2,3,4,11,12,13,14)$.

Embora o senso comum tenha "banalizado" a coqueluche como doença infantil, verifica-se o crescente contágio entre adolescentes e adultos. Estudos epidemiológicos realizados na França desde a década de 1990 demonstraram que a incidência de coqueluche permanecia elevada na Europa - 12,2/100.000 (2, 3, 9, 14).

No Brasil, não existem estudos conclusivos que possibilitem responder inequivocamente se há ou não a re-ermergência de pertussis, considerando especialmente os fatores que interferem no monitoramento da doença, tais como: a dimensão territorial do país, as variações socioeconômicas, climáticas, culturais e de infraestrutura relativa ao acesso ao sistema de saúde pública e dos sistemas de notificação. Estes aspectos relevantes fazem parte da realidade nacional e estão relacionados às possibilidades de análises do comportamento epidemiológico da doença. Contudo, algumas hipóteses podem ser levantadas. Uma delas refere-se à possibilidade de que a doença esteja de fato reemergindo no Brasil de forma não perceptível, embora, nos últimos anos, alguns estados tenham relatado um aumento significativo no número de casos $(8,11,12,14)$.

As medidas de biossegurança adotadas desde o pronto atendimento até o isolamento de pacientes suspeitos ou confirmados com coqueluche e/ou tuberculose, em especial a medida preventiva do isolamento, podem ser recomendadas. A re-emergência da coqueluche é agravada pelo fato de, hoje, pertussis não poder mais ser considerada uma doença exclusiva do campo da pediatria. Ela requer uma maior complexidade de percepção e de ações relativas ao diagnóstico, à epidemiologia e a outras especialidades que estão no âmbito da saúde pública. A Organização Mundial da Saúde e a Organização Panamericana de Saúde, em 1960, definiram o isolamento como a segregação de pessoas infectadas, durante o período de transmissibilidade da doença, em local sob condições para evitar a transmissão direta ou indireta do agente infeccioso a indivíduos suscetíveis ou que possam transmitir a outros. $(5,14)$.

No Manual para el Control de las Enfermedades Transmisibles, uma publicação da OPAS, o isolamento é referido como uma medida de controle do 
paciente, seus contatos e ambiente e inclui medidas destinadas a evitar que o indivíduo infectado siga disseminando a doença (3).

Este estudo estabeleceu como objetivo de investigação a percepção dos profissionais de saúde das unidades pesquisadas diante do atendimento a pacientes suspeitos ou confirmados com doenças respiratórias, como coqueluche e tuberculose, a adoção das práticas de biossegurança e a existência de programas de capacitação continuada nas unidades de saúde.

\section{METODOLOGIA}

Foram eleitos como locais de coleta de informações no Estado do Rio de Janeiro seis estabelecimentos de atendimento, segundo as particularidades de cada um deles: uma instituição de pesquisa que pratica atendimentos e acolhe portadores de doenças emergentes, re-emergentes e negligenciadas (unidade civil federalUCF); um hospital militar federal (HMF) de grande porte com boa infraestrutura (com número expressivo de clínicas, leitos, berçário, CTI); uma policlínica militar federal (PMF) com grande número de atendimento pediátrico; um estabelecimento de assistência pública municipal, unidade de saúde municipal I (USM-I), com grande demanda e com deficiências infraestruturais; uma instituição de saúde do interior do Estado do Rio de Janeiro também com deficiências infraestruturais, unidade de saúde municipal II (USM-II) e uma unidade de saúde da família, unidade de saúde municipal III (USM-III).

Tais informações foram obtidas por meio da aplicação de questionários previamente elaborados com base na observação das particularidades de cada unidade por meio de visitas técnicas. Nestes questionários, privilegiamos perguntas relacionadas aos critérios de isolamento de pacientes com suspeita ou confirmação de coqueluche, à adoção de medidas de biossegurança pelos profissionais de saúde em casos suspeitos ou confirmados de coqueluche e tuberculose e também à existência de programa de capacitação continuada nas unidades de saúde.

A pesquisa sobre os procedimentos de biossegurança em casos suspeitos ou confirmados de tuberculose foi adotada neste estudo como fator de comparação, pois ambas as doenças (coqueluche e tuberculose) apresentam sintomatologia e transmissibilidade semelhante na fase inicial, porém os procedimentos de biossegurança adotados para paciente suspeito ou confirmado com tuberculose são amplamente conhecidos pelos profissionais de saúde que trabalham com doenças infecto-parasitárias.

\section{RESULTADOS}

Em relação às unidades de saúde, observamos que a realidade é bem diferenciada quanto ao critério de isolamento de paciente com coqueluche: a) profissionais que realizaram isolamento em quarto com sistema de filtragem de ar 
- HMF (42\%), UCF (100\%), PMF (11\%), unidades municipais USM-I , USM-II e USM-III (não realizaram tais procedimentos); b) profissionais que realizaram isolamento em quarto sem sistema de filtragem de ar - HMC (29\%), PMF (45\%), USM-I (30\%), USM-II (14\%) e USM-III (14\%) (Tabela 1).

Tabela 1. Critério de isolamento de paciente suspeito ou confirmado com coqueluche nas unidades de saúde do Rio de Janeiro

\begin{tabular}{lcccccc}
\hline & HMF & UCF & PMF & USM-I & USM-II & USM-III \\
\hline Quarto com filtragem de ar & $42 \%$ & $100 \%$ & $11 \%$ & & & \\
\hline Quarto sem filtragem de ar & $29 \%$ & & $45 \%$ & $30 \%$ & $14 \%$ & $14 \%$ \\
\hline Enfermeria & $2 \%$ & & & & & \\
$\begin{array}{l}\text { Não realizam o isolamento por } \\
\text { falta de infraestrutura }\end{array}$ & $12 \%$ & & $11 \%$ & $30 \%$ & $57 \%$ & $57 \%$ \\
\hline Não responderam & $15 \%$ & & $33 \%$ & $40 \%$ & $29 \%$ & $29 \%$ \\
\hline
\end{tabular}

Legenda: HMF, hospital militar federal; UCF, unidade civil federal; PMF, policlínica militar federal; USM-I, unidade saúde municipal I; USM-II, unidade saúde municipal II e USM-III, unidade saúde municipal III.

Cabe destacar que $57 \%$ dos profissionais da USM-II e USM-III não realizaram o isolamento por falta de infraestrutura e $40 \%$ da USM-I não responderam a este questionamento.

Quanto aos critérios de biossegurança adotados pelos profissionais de saúde em relação a paciente suspeito ou internado com coqueluche, desde o pronto atendimento até possível isolamento, foram observados os seguintes percentuais: $32 \%$ do HMF informaram que raramente eles são adotados, $45 \%$ do UCF informaram que sempre os adotaram, 29\% do PMF frequentemente utilizaram tais procedimentos, $60 \%$ do USM-I informaram que raramente são utilizados os procedimentos, $43 \%$ do USM-II não os adotaram e $42 \%$ dos profissionais da USM-III não responderam a esta pergunta (Tabela 2).

Tabela 2. Adoção dos procedimentos de biossegurança para paciente suspeito ou confirmado com coqueluche nas unidades de saúde do Rio de Janeiro

\begin{tabular}{lcccccc}
\hline & HMF & UCF & PMF & USM-I & USM-II & USM-III \\
\hline Sempre & $23 \%$ & $45 \%$ & $17 \%$ & & & \\
\hline Maioria & & & & & $15 \%$ & \\
\hline Frequentemente & $26 \%$ & $22 \%$ & $29 \%$ & $10 \%$ & & \\
\hline Parte dos profissionais & $19 \%$ & $11 \%$ & $18 \%$ & $20 \%$ & $14 \%$ & \\
\hline Raramente & $32 \%$ & $11 \%$ & $6 \%$ & $60 \%$ & $14 \%$ & $29 \%$ \\
\hline Não utilizam os proced. biosseg. & & & & & $43 \%$ & $29 \%$ \\
\hline Não responderam & $11 \%$ & $18 \%$ & $10 \%$ & $14 \%$ & $42 \%$ \\
\hline Não se aplicam & & $12 \%$ & & & \\
\hline
\end{tabular}

Legenda: HMF, hospital militar federal; UCF, unidade civil federal; PMF, policlínica militar federal;USM-I, unidade saúde municipal I; USM-II, unidade saúde municipal II e USM-III, unidade saúde municipal III.

Fonte :Dados da pesquisa 
O questionamento sobre os mesmos procedimentos de biossegurança em relação a paciente suspeito ou confirmado com tuberculose obteve os seguintes resultados: $50 \%$ dos profissionais do HMF informaram que foram adotados por apenas parte dos profissionais, $67 \%$ do UCF informaram sua adoção pela maioria dos profissionais, $50 \%$ do PCF informaram que a adoção se deu por uma minoria, $60 \%$ do USM-I por apenas uma parte dos profissionais, $29 \%$ do USM-II não utilizaram tais procedimentos e $29 \%$ da USM-III informaram que a maioria adotou os procedimentos, porém $29 \%$ da mesma unidade relataram a adoção por uma minoria dos profissionais de saúde (Tabela 3 ).

Tabela 3. Adoção dos procedimentos de biossegurança para paciente suspeito ou confirmado com tuberculose nas unidades de saúde do Rio de Janeiro

\begin{tabular}{lrrrrrc}
\hline & HMF & UCF & PMF & USM-I & USM-II & USM-III \\
\hline Sempre & $2 \%$ & $22 \%$ & $6 \%$ & & $15 \%$ & \\
\hline Maioria & $15 \%$ & $67 \%$ & $22 \%$ & & $14 \%$ & $29 \%$ \\
\hline Parte dos profissionais & $50 \%$ & $11 \%$ & $22 \%$ & $60 \%$ & $14 \%$ & \\
\hline Raramente & $33 \%$ & & $50 \%$ & $30 \%$ & $14 \%$ & $29 \%$ \\
\hline Não utilizam os proced. biosseg. & & & & & $29 \%$ & $14 \%$ \\
\hline Não responderam & & & & $10 \%$ & $14 \%$ & $28 \%$ \\
\hline
\end{tabular}

Legenda: HMF, hospital militar federal; UCF, unidade civil federal; PMF, policlínica militar federal;USM-I, unidade saúde municipal I; USM-II, unidade saúde municipal II e USM-III, unidade saúde municipal III.

Fonte: Dados da pesquisa.

Em relação à capacitação continuada nas unidades de saúde estudadas, obtivemos estes resultados: para 63\% do HMF ela não ocorre, para 56\% do UCF existe capacitação continuada, para 76\% PMF não há este programa, para $83 \%$ da USM-I algumas vezes ocorrem capacitações, para 17\% da USM-II não existe o programa e para $86 \%$ da USM-III não foi implementado tal programa de capacitação (Tabela 4).

Tabela 4. Programa de capacitação continuada nas unidades de saúde

\begin{tabular}{lrrrrrr}
\hline & HMF & UCF & PMF & USM-I & USM-II & USM-III \\
\hline Sim & $7 \%$ & $56 \%$ & $6 \%$ & & $16 \%$ & \\
\hline Algumas vezes & $30 \%$ & $44 \%$ & $18 \%$ & $83 \%$ & $17 \%$ & $14 \%$ \\
\hline Não & $63 \%$ & & $76 \%$ & $17 \%$ & $17 \%$ & $86 \%$ \\
\hline Não responderam & & & & & $33 \%$ & \\
\hline
\end{tabular}

Legenda: HMF, hospital militar federal; UCF, unidade civil federal; PMF, policlínica militar federal;USM-I, unidade saúde municipal I; USM-II, unidade saúde municipal II e USM-III, unidade saúde municipal III.

Fonte: Dados da pesquisa. 


\section{DISCUSSÃO E CONCLUSÃO}

Nas cinco unidades estudadas, apenas a unidade civil federal informou que $100 \%$ dos doentes são internados em isolamento com sistema de filtragem de ar, as demais unidades de saúde, federais ou municipais, informaram índice inferior a $50 \%$ ou não informaram. A análise de outro sistema de bloqueio da disseminação da doença, por meio de quarto de isolamento sem filtração do ar ambiente, revelou índice abaixo de $50 \%$ dos profissionais que adotaram este procedimento nas unidades de saúde: HMF, PMF, USM-I, USM-II e USM-III.

Segundo dados registrados no Sistema Nacional de Agravos de Notificação (SINAN), até a última semana epidemiológica de 2012, foram notificados 15.428 casos suspeitos de coqueluche no país. Destes, 4.453 (28,9\%) foram confirmados, representando um incremento de $97 \%$ em relação ao mesmo período de 2011 quando foram confirmados 2.258 casos (11).

Atualmente, os indivíduos adultos e jovens adolescentes são os principais portadores assintomáticos e transmissores da doença para crianças abaixo de 1 ano de idade. Sendo assim, é fundamental a adoção de medidas de contenção na rotina das unidades de saúde para evitar a disseminação do microorganismo, principalmente entre os profissionais da área de saúde que manipulam ou tenham contato com pacientes e/ou microorganismos $(3,5,13)$. Tais medidas são de grande relevância, uma vez que evitam que estes profissionais se tornem também portadores assintomáticos e transmissores da doença, principalmente dentro de sua unidade.

Em relação à adoção dos procedimentos de biossegurança pelos profissionais de saúde desde o pronto atendimento até o isolamento para paciente suspeito ou confirmado com coqueluche, somente $45 \%$ da equipe médica da unidade civil federal informaram que os profissionais sempre adotaram os procedimentos, correspondendo ao maior índice de todas as demais unidades: HMC (23\%) e PMF (17\%).

Quando comparamos este resultado com pacientes com suspeita ou confirmação de tuberculose, somente $22 \%$ da equipe médica da UCF informaram que sempre os profissionais de sua unidade utilizam os procedimentos de biossegurança desde o pronto atendimento até o isolamento. As equipes médicas das demais unidades, em sua maioria, informaram que somente parte dos profissionais valoriza e utiliza os procedimentos de biossegurança, acentuando que uma minoria utiliza os equipamentos de proteção durante o contato com o paciente. Este contexto de risco mostra-se preocupante, especialmente diante da realidade de aumento da tuberculose e de sua complexidade. Observamos que, no ano de 2011, foram notificados 69.245 casos de tuberculose, destacando-se o aumento de notificação de casos por cepas multirresistentes ao uso de antibióticos (8).

A coqueluche é uma doença que não está diariamente na rotina de atendimento médico, provavelmente em razão do desconhecimento de sua ressurgência. Diferentemente, a tuberculose é uma doença de amplo conhecimento 
e suas práticas de biossegurança deveriam ser adotadas por todos dos profissionais de saúde rotineiramente no contato com pacientes suspeitos ou internados.

Em relação à existência de programa de capacitação continuada nas unidades de saúde, somente 56\% dos profissionais de UCF informaram que existe tal programa e a grande maioria das unidades estudadas informou não haver política de capacitação: HMF (63\%), PMF (76\%) e USM-III (86\%).

O programa de capacitação continuada nas unidades de saúde é fundamental em todos os aspectos, tanto em relação aos princípios da biossegurança para coqueluche ou tuberculose como também para a conscientização da equipe médica sobre a necessidade de isolamento de paciente com doença infectocontagiosa em ambiente hospitalar adequado. Destaca-se a necessidade de contínuo investimento na infraestrutura das unidades de saúde, principalmente nas unidades municipais que, neste estudo, apresentaram condições inadequadas de trabalho com pacientes acometidos de doenças como tuberculose e coqueluche.

Cabe ressaltar que a falta de conhecimento qualificado para estabelecer corretamente as medidas preventivas ante as doenças respiratórias, a baixa adoção de medidas de biossegurança pelos profissionais de saúde desde o pronto atendimento até o isolamento para casos suspeitos ou confirmados de coqueluche e/ ou tuberculose são indicações concretas da falta de investimento pelas unidades de saúde em recursos humanos por meio de programa de capacitação continuada para biossegurança em doenças re-emergentes. Destacamos que, em todas as unidades estudadas, não houve um índice maior que $56 \%$ para a existência de programa de capacitação continuada.

Observamos, neste estudo, que os "Indicadores de Qualidade em Biossegurança" instituídos pela Portaria $\mathrm{n}^{\mathrm{o}} 3.204$, de 20 de outubro de 2010, do Ministério da Saúde não têm sido prioridades nas instituições pesquisadas. Tais parâmetros estabelecidos pela portaria mencionada destacam a importância de se "[...] monitorar e avaliar a eficácia da implantação das políticas e ações institucionais em Biossegurança, que devem considerar, entre outras, as condições de infraestrutura predial de equipamentos, o uso de boas práticas e capacitação profissional" $(5,8)$.

Concluímos que o aprimoramento profissional nas unidades de saúde por meio de investimento em programa de capacitação continuada nas temáticas biossegurança e doenças respiratórias, como coqueluche e tuberculose, é fundamental para reverter a situação encontrada nesta pesquisa, bem como maior investimento em infraestrutura nas unidades de saúde, sobretudo nas unidades municipais.

\section{AGRADECIMENTO}

A todos os profissionais que contribuíram para a realização deste trabalho e a suas respectivas unidades de saúde. 


\section{REFERÊNCIAS}

1. American Academy of Pediatrics. Pertussis. In: Pickering LK. ed. 2000 Red Book: Report of the Committee on Infectious Diseases. $25^{\mathrm{a}}$ ed. ELK Grove Village IL. American Academy of Pediatrics 2000. p. 435-448.

2. Bégué P, Baron S, Grimprel E. Epidémiologie de la coqueluche en Europe en 1995. Médecine et Maladies Infectieuses 25: 1263-1267, 1995.

3. Benenson AS. Manual para el control de las enfermedades transmisibles / Control of communicable diseases. Manual. Washington, Organización Panamericana de la Salud, 1997.

4. Bisgard KM, Pascual FB, Ehresmann KR, Miller CA, Cianfrini C, Jennings CE, Rebmann CA, Gabel J, Schauer SL, Lett SM. Infant pertussis: who was the source? Pediatr Infect Dis $J$ 23: 985-989, 2004.

5. BRASIL. Ministério da Saúde. Portaria No 3.204, de 20 de outubro de 2010. Aprova Norma Técnica de Biossegurança para Laboratórios de Saúde Pública. [acessed 03/01/2013]. http://bvsms.saude. gov.br/bvs/saudelegis/gm/2010/prt3204_20_10_2010.htm

6. Czeresnia D. Do contágio à transmissão: ciência e cultura na gênese do conhecimento. Rio de Janeiro, Ed. Fiocruz, 1997.

7. Forsyth KD, Wirsing VKCH, Tan T, Caro JS. Prevention of pertussis: recommendations derived from the second Global Pertussis Initiative round table meeting. Vaccine 25: 2634-2642, 2007.

8. Ministério da Saúde. Manual das doenças infecciosas e parasitárias.8 ed [acessed 02/10/2013[http:// bvsms.saude.gov.br/bvs/publicacoes/doencas_infecciosas_parasitaria_guia_bolso.pdf]

9. Nichiata LY, Gir E, Takahashi RF, Ciosak SI. Evolução dos isolamentos em doenças transmissíveis: os saberes na prática contemporânea. Rev Enferm USP 38: 61-70, 2004.

10. Nightingale F. Notas sobre enfermagem: o que é e o que não é. São Paulo: Cortez; 1989.

11. Sistema Nacional de Agravo de Doenças. Ministério da Saúde. [acessed 02/10/2013]http://dtr2012. saude.gov.br/sinanweb/tabnet/dh?sinannet/coqueluche/bases/coquebrnet.def

12. Trevizan S, Coutinho SED. Perfil epidemiológico da coqueluche no Rio Grande do Sul, Brasil: estudo da correlação entre incidência e cobertura vacinal. Cad Saúde Pública 24: 93-102, 2008.

13. Wendelboe AM, Njamkepo E, Bourillon A, Floret DD, Gaudelus J, Gerber M, Grimprel E, Greenberg D, Halperin S, Liese J, Muñoz-Rivas F, Teyssou R, Guiso N, Van Rie A. Infantil Coqueluche Group of Study. Transmission of Bordetella pertussis to young infants. Pediatr Infect Dis J 26: 293-299, 2007.

14. WHO-recommended surveillance standard of pertussis. [acessed 02/05/2013].http://www.who.int/ immunization_monitoring/diseases/pertussis_surveillance/en/index.html 